\title{
Philosophiques
}

\section{Brandom et les sources de la normativité}

\section{Joseph Heath}

Volume 28, numéro 1, printemps 2001

\section{La nature des normes}

URI : https://id.erudit.org/iderudit/004933ar

DOI : https://doi.org/10.7202/004933ar

Aller au sommaire du numéro

\section{Éditeur(s)}

Société de philosophie du Québec

\section{ISSN}

0316-2923 (imprimé)

1492-1391 (numérique)

Découvrir la revue

\section{Citer cet article}

Heath, J. (2001). Brandom et les sources de la normativité. Philosophiques, 28(1), 27-46. https://doi.org/10.7202/004933ar

\section{Résumé de l'article}

Robert Brandom a tenté de déplacer le concept de représentation de sa position de concept explicatif central en philosophie du langage et de le remplacer par un ensemble de concepts explicatifs dérivés de l'analyse de l'action sociale. Il soutient que le concept de norme sociale peut servir de concept primitif dans le développement d'une théorie générale de la signification. Selon Brandom, le problème central lié au fait de considérer la représentation comme un primitif explicatif est que nous n'avons pas une compréhension claire de ce à quoi correspond la relation de "représentation ". Il est donc naturel de s'attendre à ce que Brandom utilise, dans son analyse de l'action sociale, des primitifs explicatifs qui sont, d'une certaine manière, moins mystérieux. En particulier, on s'attend à ce que Brandom démontre que le concept de " norme sociale " peut être compris en termes d'un ensemble plus simple de concepts issus de la philosophie de l'action. Malheureusement, Brandom ne fournit pas une telle explication. Dans cet article, je commence par analyser l'argument proposé par Brandom, et je tente d'expliquer pourquoi cet argument, en définitive, n'est pas concluant. J'essaie ensuite de développer l'explication des origines de la normativité dans l'action sociale que, selon moi, Brandom aurait dû donner.
Ce document est protégé par la loi sur le droit d'auteur. L’utilisation des services d’Érudit (y compris la reproduction) est assujettie à sa politique d'utilisation que vous pouvez consulter en ligne.

https://apropos.erudit.org/fr/usagers/politique-dutilisation/ 


\title{
Brandom et les sources de la normativité
}

\author{
JOSEPH HEATH \\ Université de Toronto \\ joseph.heath@utoronto.ca
}

\begin{abstract}
RÉSUMÉ. - Robert Brandom a tenté de déplacer le concept de représentation de sa position de concept explicatif central en philosophie du langage et de le remplacer par un ensemble de concepts explicatifs dérivés de l'analyse de l'action sociale. Il soutient que le concept de norme sociale peut servir de concept primitif dans le développement d'une théorie générale de la signification. Selon Brandom, le problème central lié au fait de considérer la représentation comme un primitif explicatif est que nous n'avons pas une compréhension claire de ce à quoi correspond la relation de « représentation ». Il est donc naturel de s'attendre à ce que Brandom utilise, dans son analyse de l'action sociale, des primitifs explicatifs qui sont, d'une certaine manière, moins mystérieux. En particulier, on s'attend à ce que Brandom démontre que le concept de «norme sociale » peut être compris en termes d'un ensemble plus simple de concepts issus de la philosophie de l'action. Malheureusement, Brandom ne fournit pas une telle explication. Dans cet article, je commence par analyser l'argument proposé par Brandom, et je tente d'expliquer pourquoi cet argument, en définitive, n'est pas concluant. J'essaie ensuite de développer l'explication des origines de la normativité dans l'action sociale que, selon moi, Brandom aurait dû donner.
\end{abstract}

ABSTRACT. - Robert Brandom has tried to displace the concept of representation from its position as the central explanatory concept in the philosophy of language, and to replace it with a set of explanatory concepts derived from the analysis of social action. He argues that the concept of a social norm can serve as a primitive concept in the development of a general theory of meaning. The central problem with taking representation as primitive, in Brandom's view, is that we have no clear understanding of what the "representation" relation amounts to. This leads one to expect that Brandom's analysis of social action will draw upon explanatory primitives that are somehow less mysterious. In particular, one expects Brandom to show that the concept of a "social norm" can be explained in terms of some simpler set of action-theoretic concepts. Unfortunately, Brandom does not provide such an explanation. In this paper, I begin by providing an analysis of the argument that Brandom does provide, and attempt to explain why it is ultimately inconclusive. I then try to articulate the account of the origins of normativity in social action that I think Brandom should have provided.

Une des sections les plus insatisfaisantes du livre très complexe et difficile de Brandom, Making it Explicit, est, malheureusement, le tout premier chapitre. ${ }^{1}$ L'objectif général de Brandom dans cet ouvrage est de déplacer le concept de représentation de sa position de concept explicatif central en

1. Brandom, Robert, Making it Explicit, Cambridge (MA), Harvard University Press, 1994. Les références subséquentes apparaissent dans le texte.

PHILOSOPHIQUES 28/1 — Printemps 2001, p. 27-46 
philosophie du langage et en épistémologie, et de le remplacer par un ensemble de concepts explicatifs dérivés de l'analyse de l'action ou de la pratique sociale. Plus spécifiquement, il veut montrer que le concept d'une norme sociale - une règle qui détermine, implicitement ou explicitement, si une action est correcte ou incorrecte - peut servir de concept primitif dans le développement d'une théorie générale de la signification. La réussite d'une telle entreprise constituerait donc une défense de certaines des intuitions fondamentales à la base du pragmatisme philosophique.

Selon Brandom, le problème central lié au fait de considérer la représentation comme un primitif explicatif est que personne n'a jamais été capable d'expliquer à quoi correspond la relation de "représentation ». Descartes, par exemple, "ne réussit manifestement pas à rendre compte ni de la nature des contenus représentationnels - en quoi consiste la représentativité des représentations - ou de ce qu'est saisir ou comprendre un tel contenu »(p. 6). Il est effectivement difficile d'expliquer la représentation sans faire appel à d'autres ressources conceptuelles plus sophistiquées. En particulier, la représentation semble posséder une dimension normative irréductible. Par exemple, la représentation n'est pas simplement une covariance causale. Représenter quelque chose est le représenter correctement. Lorsque la comparaison trompeuse avec la perception visuelle est écartée, la représentation semble dès lors constituer un très mauvais candidat au rôle de primitif explicatif.

Cette critique du représentationalisme mène fort naturellement le lecteur à croire que la préférence de Brandom pour le pragmatisme repose sur le sentiment que les primitifs explicatifs disponibles dans ce cadre sont en quelque sorte moins mystérieux, ou plus intuitivement accessibles. En particulier, on s'attend à ce que Brandom démontre que le concept de "norme sociale » sur lequel il appuie son analyse du langage peut, à son tour, être compris en termes d'un ensemble plus simple de concepts béhavioristes ou issus de la philosophie de l'action. Et, comme c'est une préférence pour un ordre pragmatique d'explication qui motive l'ensemble du projet de Brandom, il ne serait pas déraisonnable de s'attendre à ce qu'une analyse des primitifs action-théorétiques soit omniprésente au début du livre. Plus encore, Brandom entreprend son premier chapitre en semblant vouloir fournir une telle analyse. Par conséquent, l'absence de tout argument ou analyse concluants à ce sujet a quelque chose de surprenant. De nombreux lecteurs de Making it Explicit terminent le premier chapitre en ne sachant pas vraiment si Brandom a choisi de ne pas traiter l'argument, s'il a proposé un argument, mais un très faible, ou s'il a choisi de reporter le fardeau de la preuve jusqu'au chapitre huit.

Cette confusion est regrettable. Comme le note Brandom, si on est prêt à accepter le concept de représentation comme donné, alors il existe une série de mécanismes bien connus qui peuvent être utilisés afin de générer, à partir de là, une explication de la vérité et de l'inférence, une théorie de l'action rationnelle, et ainsi de suite. L'essentiel de Making it Explicit consiste en la tentative de Brandom de montrer que, si on est prêt à accepter le concept 
d'une norme sociale comme donné, on peut de la même manière générer une explication de l'inférence, de la vérité, de la référence et, ultimement, de la représentation. Toutefois, le mécanisme utilisé pour atteindre ce but demeure relativement inexploré et chargé de difficultés techniques. Afin qu'il vaille la peine de résoudre les difficultés inhérentes à ce mécanisme, Brandom doit fournir au lecteur une raison de penser qu'il est en quelque sorte plus plausible de considérer la normativité, plutôt que la représentation, comme un primitif. Toutefois, le premier chapitre de Making it Explicit n'y parvient manifestement pas.

Le but de cet article est double. Premièrement, j'analyserai l'argument que Brandom propose dans le premier chapitre et développerai une hypothèse visant à expliquer pourquoi cette analyse demeure si peu probante. Mon deuxième but est plus direct : je développerai l'explication des origines

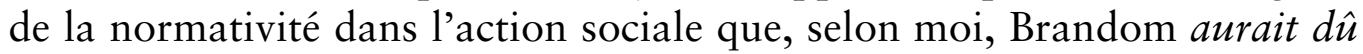
fournir (en fait, qu'il a presque fournie).

1.

L'analyse que fait Brandom des normes sociales est tributaire de sa tentative d'éviter deux stratégies explicatives qu'il considère infructueuses. La première de ces stratégies, que Brandom appelle régulisme, identifie les normes avec une sorte de formulation explicite d'une règle. Ainsi, on comprendra une norme sociale par analogie avec, par exemple, une affiche sur la plage disant " pas de baignade ». L'évaluation normative d'une action est possible parce qu'on peut prendre une action particulière, la comparer avec une règle précisant comment l'action doit être accomplie, et déterminer si cela a été fait correctement ou incorrectement. Selon Brandom, cette approche est infructueuse, parce que l'espèce pertinente de normativité n'est que subsidiaire ou dérivée. "Les propriétés d'exécution qui sont régies par des règles explicites ne forment pas une strate autonome de statuts normatifs, une strate qui pourrait exister alors qu'aucune autre n'existerait» (p. 20).

Le problème inhérent à ce type de régularisme a été clairement identifié par Kant, mais a été formulé de façon plus incisive par Wittgenstein. L'objection de base prend la forme d'un argument de régression. Une règle explicite ne peut, par elle-même, déterminer le statut normatif d'autre chose. Elle doit être appliquée. Mais l'application est aussi quelque chose qui peut être fait correctement ou incorrectement. Par conséquent, il doit y avoir une norme de second ordre qui précise la façon dont les normes de premier ordre doivent être appliquées. Mais alors, comment ces normes de second ordre doivent-elles être appliquées ? Il doit y avoir une norme de troisième ordre qui précise la façon dont elles doivent être appliquées. Une régression vicieuse s'ensuit.

Quelle leçon doit-on tirer de cet argument ? Selon Wittgenstein, « ce que cela montre est qu'il y a une manière de saisir une règle qui n'est pas une interprétation, mais qui se manifeste par ce que nous appelons "obéir à la 
règle" et "ne pas lui obéir" dans des cas précis. $»^{2} \mathrm{Ou}$, comme le dit Brandom, " il y a un besoin pour une conception pragmatiste des normes - une notion des convenances primitives de l'exécution implicites dans la pratique qui sont présupposées par et précèdent leur formulation explicite dans des règles et des principes »(p. 21). La régression survient en raison de la croyance que les normes résident originairement dans des "principes ", et sont appliquées aux "pratiques » seulement de façon secondaire. Le problème est alors que l'on ne peut jamais passer des principes aux pratiques, d'autres principes semblant toujours être requis. Par conséquent, la solution consiste à formuler une explication de ce que signifie qu'il existe des normes implicites dans les pratiques.

Ces considérations conduisent à un certain anti-intellectualisme au sujet des normes. L'argument de Wittgenstein nous rappelle de ne pas confondre les règles elles-mêmes avec nos formulations explicites ou idéationelles de celles-ci. Mais, bien que ce rappel soit utile, il est possible de pousser trop loin cet anti-intellectualisme et de présumer que les règles peuvent être identifiées d'une manière qui néglige complètement nos attitudes envers elles. Une telle approche consisterait à assimiler les normes sociales à de simples régularités dans la conduite. Cette idée est au cœur du second type de stratégie explicative, stratégie à laquelle Brandom fait référence en utilisant le terme de régularisme.

Selon Brandom, le problème avec le régularisme est qu'il perd de vue la distinction entre ce qui est fait et ce qui doit être fait. En d'autres mots, il perd de vue la dimension proprement normative des normes sociales. Un symptôme de cette difficulté - et une objection cruciale au régularisme - est le problème du "découpage arbitraire ". Pour un ensemble fini de comportements, on peut imaginer un nombre arbitrairement grand de règles desquelles ce comportement serait une exemplification. Par conséquent, lorsque nous sommes confrontés à une forme de comportement qui semble dévier d'une règle, il est toujours possible de créer une autre règle avec laquelle ce comportement serait cohérent.

L'erreur du régulariste consiste à penser que, simplement parce que les normes ne consistent pas en nos représentations explicites de celles-ci, nos attitudes doivent être complètement éliminées de l'explication. Ainsi, le régulariste espère découvrir la présence de normes simplement en étudiant les régularités de comportement, tout en ignorant complètement la question de savoir ce que les agents croient eux-mêmes faire. C'est ultimement cela qui génère le problème du "découpage arbitraire ". Si l'on ne porte pas attention à ce que les agents croient eux-mêmes être en train de faire, il y aura simplement trop de règles qui « seront cohérentes avec » le cas concret. De plus,

2. Wittgenstein, Ludwig, Philosophical Investigations, trad. G. E. M. Anscombe, Oxford, Basil Blackwell, 1953, \$201, p. 81e. 
toute chose est l'exemplification d'une quelconque règle. Ainsi, la distinction entre un acte correct et incorrect s'écroule.

Selon Brandom,

afin que la simple identification régulariste de l'impropriété avec l'irrégularité soit convaincante, elle doit être complétée par une manière de choisir, comme si elles étaient d'une certaine manière privilégiées, certaines régularités parmi celles qui sont présentées. Affirmer cela, c'est affirmer que certaines régularités doivent être choisies comme étant celles qui doivent être observées, certaines structures comme étant celles qui doivent être perpétuées. Dans sa forme la plus simple, l'approche de la régularité n'offre aucune suggestion quant à savoir comment cela pourrait être fait et, ainsi, ne résout pas le problème mais déplace seulement la question de savoir comment comprendre la distinction normative entre ce qui est fait et ce qui doit être fait (p. 28).

L'astuce consiste donc à trouver une manière d'agir qui soit justement comprise comme un exemple du fait de " considérer quelque chose comme étant correct »- exprimant ainsi la bonne sorte d'attitude normative mais qui ne soit pas elle-même une formulation explicite de l'idée qu'une chose ou une autre est correcte. Ainsi, afin de trouver des normes implicites en pratique, nous devons trouver des évaluations normatives de l'action implicite dans la pratique.

\section{2.}

Le candidat le plus évident pour un type de comportement manifestant une évaluation normative est la sanction. Nous répondons à des actions qui sont correctes par des sanctions positives, à des actions incorrectes par des sanctions négatives. Une sanction positive peut être ici comprise comme toute chose possédant un statut gratifiant pour l'agent sur lequel on agit et qui, par conséquent, renforce le comportement - une récompense. Une sanction négative est toute chose qui possède une signification négative et qui, par conséquent, conditionne l'agent à ne pas répéter le comportement - une punition.

La manière la plus directe de générer une explication des normes sociales à partir de cette conception de la sanction est simplement de définir une norme comme étant une régularité sanctionnée dans la conduite. Brandom prête une théorie de ce type à John Haugeland. Selon une telle conception, les agents se conforment à certaines structures parce qu'elles sont positivement sanctionnées, ou parce que tout écart est négativement sanctionné, ou pour les deux raisons. Ainsi, leurs actions sont implicitement sujettes à une évaluation normative - une action est implicitement jugée correcte lorsqu'elle entraîne une sanction positive, et incorrecte lorsqu'elle entraîne une sanction négative. Cette sanction est ce qui privilégie une structure particulière, l'élevant au-dessus de la simple régularité.

Cette explication a quelque chose de séduisant du fait que la sanction peut être comprise sans avoir à présupposer d'autres concepts normatifs alors 


\section{Philosophiques / Printemps 2001}

que, pourtant, elle compte clairement comme un type d'évaluation normative implicite. Néanmoins, Brandom la considère comme inadéquate. Sa principale inquiétude tient au fait qu'il s'agit toujours d'un type de théorie régulariste qui, par conséquent, "ne fait que reporter le problème du découpage arbitraire ". L'introduction de sanctions permet d'identifier une structure privilégiée à la base même du comportement. Mais l'acte de sanctionner lui-même est simplement une autre structure de comportement, et peut donc être compris comme «faisant respecter » un nombre arbitraire de règles différentes.

Tout comme il n'existe pas une telle chose que la régularité de l'acte qui soit manifestée par une certaine manière d'agir réelle (...), il n'existe pas une telle chose que la régularité qui soit renforcée par un certain ensemble de réponses à des réponses, ou même de dispositions à répondre à des réponses. Le problème du "découpage arbitraire ", soit de la manière de privilégier une spécification de régularité parmi les autres compétiteurs également qualifiés, surgit encore une fois au niveau de la régularité renforçante. (p. 36)

Une façon de présenter le problème serait de dire que, selon la conception élémentaire de la sanction, il est impossible de déterminer si la personne qui sanctionne fait un «bon travail». Les actions de celui qui sanctionne constituent simplement une structure de comportement, qui peut elle aussi être comprise comme exemplifiant une certaine règle. Ainsi, la distinction entre " ce qui doit être fait » et " ce qui est fait " disparaît à ce niveau.

Une manière évidente de tenter de régler ce problème serait d'ajouter un autre niveau de sanctions - de traiter l'acte de sanctionner comme étant lui-même sujet à de nouvelles sanctions. Cela équivaudrait à reconnaître qu' "évaluer, sanctionner, est en soi quelque chose qui peut être fait correctement ou incorrectement »(p. 36). Toutefois, cette stratégie ne fait que reporter le problème du " découpage arbitraire » et, ultimement, elle génère une régression. Peu importe le nombre de "niveaux » de sanctions qui sont introduits, il y aura toujours de l'arbitraire dans la structure au « plus haut » niveau. Ainsi, selon Brandom, "si le renforcement réel de régularités dispositionnelles est la seule chose disponible pour expliquer cette régression, on peut toujours affirmer que ce qui est institué par cette hiérarchie de régularités de réponses à des régularités de réponses ne doit pas être considéré comme authentiquement normatif ${ }^{3} »(\mathrm{p} .36)$.

(Soit dit en passant, on ne peut pas non plus rapiécer cette explication en se tournant simplement vers les régularités de l'évaluation commune. Brandom observe que le problème du " découpage arbitraire » demeure, que ce soit une personne ou un groupe de personnes qui soit impliqué dans l'évaluation.)

3. Brandom utilise une phrase bizarrement non-compromettante : «pourrait toujours être affirmé ». Sa signification n'est pas claire. 
3.

Quelle est alors la solution de Brandom ? De son point de vue, la réponse implique de s'éloigner de l'idée selon laquelle l'acte de sanctionner doit être compris en "termes naturalistes »(p. 42). Lorsque les sanctions sont comprises en termes de récompenses et de punitions, le but est clairement d'expliquer les évaluations normatives en termes d'un ensemble d'actions qui peuvent, elles, être comprises en termes non-normatifs. Mais, selon Brandom, « l'engagement envers une telle réduction est optionnel »(p. 43).

Brandom reconnaît qu'une manière de sanctionner quelqu'un est de faire quelque chose qui possède une signification intrinsèque gratifiante ou punitive pour cette personne. Toutefois, une punition peut aussi consister dans un changement de statut normatif. Accomplir correctement une action peut avoir un effet sur toute une gamme d'actions qu'on peut subséquemment être en droit de faire. Accomplir incorrectement une action peut rendre incorrect de tenter une autre action donnée. Ainsi, la sanction en réponse à une action peut n'être rien d'autre qu'un changement de statut normatif. Comme Brandom l'exprime :

Comme il a été noté plus haut, la réponse évaluative constituant la reconnaissance par la communauté d'une telle norme (l'attitude correspondant au statut) peut, dans certains cas, se décrire en termes non-normatifs - celui qui viole la norme est battu avec des bâtons, le comportement violant la norme est négativement sanctionné. Mais d'autres cas sont possibles, par exemple ceux dans lesquels la réponse évaluative est de punir en rendant d'autres actions inappropriées - celui qui viole la norme n'est pas en droit d'assister au festival hebdomadaire. Dans un tel cas, la signification normative de la transgression est elle-même spécifiée en termes normatifs (de ce qui est approprié, de ce que le transgresseur est en droit de faire)(p. 43).

Brandom dit d'une sanction qui ne fait que changer le statut normatif qu'il s'agit d'une «sanction interne » (puisque sa force est interne au système normatif), et d'une sanction de la variété du battre-avec-un-bâton qu'il s'agit une "sanction externe».

Évidemment, l'introduction de sanctions internes ne changera absolument rien à l'ensemble de l'explication si ces sanctions ne servent que de succédanés aux sanctions externes. On pourrait argumenter que ce ne sont que les sanctions externes qui comptent "vraiment ", et que chaque chaîne de sanctions internes doit, en bout de ligne, être " ancrée » dans quelques sanctions externes. Alors qu'on pourrait ne pas être battu avec un bâton mais seulement être banni du festival pour, par exemple, avoir manqué la chasse, on sera battu avec des bâtons si on essaie subséquemment d'assister au festival. Mais alors, tout cela peut être décrit à nouveau de manière à ce que toute référence à la sanction interne soit évitée seulement en disant que celui qui manque la chasse et tente d'assister au festival sera battu avec des bâtons. De cette façon, ce type de construction ne serait pas, essentiellement, diffé- 


\section{Philosophiques / Printemps 2001}

rent de celui de Haugeland. La sanction externe demeure celle qui fait tout le travail, de telle sorte que le problème du "découpage arbitraire " de la structure sous-jacente à ces sanctions persiste.

Par conséquent, la distance cruciale que prend Brandom par rapport au modèle de Haugeland réside dans son affirmation que les sanctions internes n'ont pas besoin d'être ultimement ancrées dans des sanctions externes (la restriction "peut être relâchée », comme il l'exprime). Il soutient qu'il n'y a rien d'incohérent dans un système normatif dont toutes les sanctions sont internes. «Une telle interprétation n'a pas besoin de supporter une réduction de l'état normatif à des dispositions non-normativement spécifiables » (p. 44). Le système serait «tout en normes »(p. 44).

Malheureusement, Brandom dit peu de choses au sujet de cette proposition. Cela est très déconcertant étant donné que l'ensemble de son projet dépend de sa capacité à expliquer l'idée voulant qu'il puisse y avoir des «normes implicites dans les pratiques ». Brandom n'explique pas exactement comment le fait d'avoir un ensemble entièrement internalisé de sanctions nous éloigne de l'explication régulariste. Mais, de façon plus importante, il ne dit presque rien afin de dissiper le doute concernant la possibilité qu'un système de normes puisse n'être constitué que de normes internes. Toutefois, aussitôt que certaines sanctions externes sont introduites, il existe un danger que l'ensemble de l'explication s'effrite et nous renvoie à celle de Haugeland.

La raison pour laquelle on pourrait être sceptique face à l'idée d'un système de sanctions entièrement internalisé est qu'il ne pourrait fonctionner que sous des conditions de très stricte obéissance. En particulier, les personnes qui sont punies devraient toujours «accepter » leurs punitions. Si une personne contrevient à une règle, elle ne sera pas directement corrigée, mais perdra simplement d'autres droits qu'elle possède dans la communauté. Mais cette perte ne serait pas directement imposée. Simplement, une prescription normative dirait : "Vous n'êtes plus en droit de faire $x$. " L'agent serait libre de désobéir à cette règle, comme il a désobéi à la première. Si toutes les sanctions sont internes, la seule manière dont les autres peuvent répondre à ce second acte de déviance est d'imposer un autre changement de statut normatif, que l'agent pourra encore une fois choisir d'ignorer. Sans un quelconque type de sanctions externes de "dernier recours ", aucune pratique ne pourrait résister à une déviance résolue.

Un autre problème apparaît lorsqu'il s'agit d'expliquer comment une communauté peut intégrer de nouveaux membres dans un ensemble de pratiques, si toutes les sanctions régissant ces pratiques sont internes. Il faudrait alors montrer qu'une pratique qui est " tout en normes " peut aussi être apprise. C'est une caractéristique évidente de la manière dont nous initions les enfants, par exemple, à nos pratiques, en recourant assez souvent aux sanctions afin de signaler notre approbation ou notre désapprobation. Ces sanctions impliquent souvent la coopération ou le refus de coopération - les parents disent toujours des choses comme "non, je ne te le donnerai pas 
avant que tu le demandes gentiment ». Ce sont des sanctions externes. Il est difficile d'imaginer que nous puissions nous passer entièrement de sanctions de ce genre, ou que les pratiques dans lesquelles elles sont utilisées ne sont pas " authentiquement » normatives à cet égard.

\section{4 .}

Étant donné ces objections évidentes à l'idée d'une pratique entièrement régie par des sanctions internes, on se demande ce que Brandom croit avoir démontré au terme de cette discussion. Je soutiens que la meilleure manière d'évaluer cela est de voir que Brandom, en suggérant qu'une pratique peut être " tout en normes ", n'essaie pas tellement de fournir une explication des fondements de la normativité en termes de théorie de l'action, mais plutôt d'offrir un refus de principe pour fournir une telle explication.

Selon Brandom, la motivation d' "expliquer » la normativité en termes de quelque chose de plus fondamental est guidée par la volonté (en définitive inopportune) de rendre les normes "respectables du point de vue naturaliste ". Selon la conception naturaliste typique, les jugements descriptifs ne sont pas terriblement mystérieux. Ils sont à propos du monde. Ils sont corrects seulement dans les cas où le monde est comme ils affirment qu'il est. D'autre part, les jugements prescriptifs - les jugements qui contiennent un " devoir-être » - sont beaucoup plus mystérieux. Nous ne savons pas vraiment ce qu'ils concernent, et nous ne savons pas comment décider s'ils sont corrects ou incorrects.

En somme, pour donner un sens aux normes, nous devons fournir une sorte de réduction. Nous avons à les expliquer en termes de quelque phénomène empirique ou " naturel ", tel le comportement. Ainsi, ce qui engendre le dilemme pour le régulariste - que ce soit la théorie régulariste de base, ou le régularisme plus sophistiqué basé sur les sanctions - est son « engagement envers la possibilité d'une réduction du normatif au dispositionnel »(p. 46). Lorsque Brandom dit qu'une pratique peut être " tout en normes ", il suggère qu'aucune réduction semblable n'est possible, ou nécessaire.

Brandom est confortable avec une position anti-réductionniste face au normatif parce qu'il pense que le concept du «naturel » est porteur d'engagements qui sont ultimement tout aussi mystérieux que le concept du normatif. En particulier, nous prenons le "monde naturel » pour une série d'événements reliés entre eux par des relations causales. Selon Brandom, ces relations causales ne peuvent être comprises qu'en termes de modalités aléthiques (nécessité, possibilité, impossibilité). Par exemple, il fait partie intégrante des relations causales qu'elles fondent des inférences menant à des contrefactuels. Brandom partage avec Sellars l'idée que ces propriétés causales sont constitutives du concept d'une large gamme de propriétés physiques, et ainsi il pense que même des descriptions simples contiennent des engagements envers des affirmations qui ne peuvent être exprimées qu'en utilisant un vocabulaire modal (p. 103). 


\section{Philosophiques / Printemps 2001}

Ainsi, le naturaliste se sert en fait des modalités aléthiques en formulant ses affirmations, même s'il est très douteux que ces modalités puissent être exprimées en termes strictement naturalistes. Par conséquent, ce que Brandom fait n'est pas plus mal. Plutôt que de se servir des modalités aléthiques, il se sert des modalités déontiques (obligatoire, permis, défendu - ou quelque chose comme cela $)^{4}$.

Bien entendu, cet argument montre seulement que Brandom n'est pas pire que le naturaliste. Mais qu'est-ce qui, au départ, pourrait lui donner le droit d'utiliser ces modalités ? La réponse à cette question se trouve dans la reconnaissance que le concept de normativité, avec les modalités déontiques, constituent des pièces du vocabulaire expressif. Ils sont des mots que nous, créatures utilisant le langage, introduisons afin de parler, en partie, des pratiques qui constituent nos compétences linguistiques. Tous deux, Brandom et le naturaliste, introduisent des formes rivales de vocabulaire expressif. Qu'un vocabulaire soit meilleur qu'un autre doit être déterminé, non par sa correspondance aux faits, mais par le niveau de "complétude expressive » (p. 641) qu'il est capable d'atteindre.

Par conséquent, la question de savoir si nous devons considérer les modalités aléthiques ou déontiques comme primitives doit être résolue par la qualité de l'histoire que nous réussissons à conter en utilisant l'un ou l'autre des ensembles de ressources expressives. Brandom tente de prouver que, moyennant l'acceptation des modalités déontiques, il est capable d'expliquer l'émergence de l'objectivité et de la représentation et d'expliquer pourquoi nos affirmations au sujet du monde objectif supportent des affirmations qui sont mieux formulées en termes de modalités aléthiques. Le naturaliste, d'autre part, même s'il se sert explicitement des modalités aléthiques, demeure incapable de donner une reconstruction adéquate des modalités déontiques.

À l'arrière plan de tout cela se trouve la conviction de Brandom que nous saisissons implicitement ce qu'est suivre une norme, parce que c'est ce que nous faisons à chaque fois que nous affirmons quelque chose. Ainsi, il n'a pas à expliquer au lecteur en quoi consiste le fait d'accomplir un acte correctement ou incorrectement. Si le lecteur ne comprenait pas déjà cela implicitement, il serait incapable de comprendre le texte. Par conséquent, le but de Brandom est expressif - il consiste à expliquer la structure du langage de l'intérieur. C'est pourquoi il n'a pas à fournir une explication «fondationnelle » de ce que sont les normes ou d'où elles proviennent. On peut donc répondre très simplement à la grande question à la base de son enquête, "Où sont les normes ? ": "Les normes sont... ici »(p. 649).

En définitive, Brandom pense que le prescriptif est plus fondamental que le descriptif. La normativité est quelque chose que nous saisissons directement.

4. Cette remarque s'explique par le fait que Brandom considère que les pièces fondamentales du vocabulaire déontique sont l'engagement et le droit à, et non l'obligation et la permission. 
Nous devons en avoir une compréhension implicite afin de seulement commencer à utiliser le langage. L'idée d'un monde naturel, contenant des objets possédant des propriétés, reliés entre eux par des relations causales, est quelque chose qui vient beaucoup plus tard. Il s'agit d'une des plus importantes réalisations de notre système linguistique, et non une présupposition. Ainsi, tout système philosophique qui tente d'en faire un primitif explicatif est condamné à demeurer profondément aporétique.

\section{$5 \cdot$}

Ceux qui connaissent l'œuvre de Brandom auront évidemment remarqué que la discussion précédente porte à l'avant-scène la dimension " hégélienne » de ses conceptions philosophiques. Un des inconvénients de cet hégélianisme réside dans la tendance générale à penser que les affirmations philosophiques ne peuvent être évaluées ponctuellement, pour ainsi dire, mais doivent être acceptées ou rejetées au niveau de «systèmes » entiers. Certainement, un des problèmes de l'argument de Brandom concernant la normativité réside dans le fait que son explication des normes sociales se tient ou s'écroule seulement avec la « complétude expressive » de sa théorie entière. (C'est aussi ce qui produit l'impression que Brandom se conçoit lui-même, au chapitre premier, comme reportant le fardeau de la preuve).

Toutefois, on ne peut s'empêcher de se demander si la stratégie argumentative de Brandom ne contiendrait pas un élément d' "espoir déçu ». Après tout, alors que Brandom peut ne pas considérer le monde physique comme un donné non-problématique, il partage avec Sellars le point de vue selon lequel quelque chose comme des dispositions réactionnelles conditionnées à l'environnement peuvent et doivent être considérées comme primitives (cela est central à son explication de l'observation). Par conséquent, on ne peut s'empêcher de penser que, si une simple explication béhavioriste de ce qu'est suivre une norme devait être disponible, une explication qui constituerait un compromis acceptable entre le régulisme et le régularisme, Brandom serait plus que content de l'adopter - et de déplacer une partie de l'emphase mise sur une dépendance à l'exhaustivité expressive comme mesure évaluative.

Une stratégie afin de développer une telle explication est suggérée par le parallèle que fait souvent Brandom entre interpréter un comportement comme intentionnel et interpréter des pratiques comme régies par des normes. En adoptant une "position intentionnelle " envers un organisme, on choisit en fait d'utiliser un certain vocabulaire en décrivant ses actions. Les éléments fondamentaux de ce vocabulaire sont les concepts de croyance et de désir. Ainsi, en adoptant un point de vue intentionnel, on choisit d'expliquer les actions des organismes comme dirigées vers un but et comme guidées par un certain ensemble de représentations relatives à l'atteinte de ce but.

Une théorie proposant d'expliquer l'intentionnalité en termes d'attribution de ces états demeure incomplète, toutefois, tant qu'elle ne traite pas 


\section{Philosophiques / Printemps 2001}

du système qui fait l'attribution. On est tenté de penser que tout système qui peut adopter un point de vue intentionnel envers un autre doit lui aussi être un système intentionnel ${ }^{5}$. Pour cette raison, Brandom distingue les systèmes intentionnels "simples" des systèmes intentionnels "interprétants ». Mais d'où provient cette intentionnalité "interprétante »? Si on postule un système intentionnel supplémentaire, dont les attributions constituent l'intentionnalité du système interprétant, on initie alors une régression. Ce qui est nécessaire pour résoudre la régression d'une manière satisfaisante, est une espèce de source de toute cette intentionnalité. Nous avons besoin d'une sorte d'intentionnalité originaire.

La façon la plus mécanique de résoudre cette régression serait d'abandonner la «posture interprétative » et de postuler un système intentionnel dont l'intentionnalité ne serait pas héritée d'un autre système. John Searle utilise un tel argument pour défendre sa conception selon laquelle certains systèmes doivent posséder une intentionnalité intrinsèque. Toutefois, Brandom veut maintenir son allégeance à la "posture interprétative ». Il rejette la tactique de Searle, choisissant plutôt d'argumenter que l'intentionnalité originaire peut être trouvée, en gros, dans les groupes où les agents s'attribuent des états intentionnels les uns aux autres. Il affirme cette position dans les termes suivants :

La clé de cette interprétation est qu'une interprétation de cette sorte doit interpréter les membres de la communauté comme se concevant ou se traitant, dans la pratique, comme adoptant des engagements intentionnellement pleins et d'autres statuts normatifs. Selon cette interprétation, si les pratiques attribuées à la communauté par le théoricien ont la structure correcte, alors les attitudes pratiques des membres de la communauté instituent des statuts normatifs et leur confèrent un contenu intentionnel ; selon cette interprétation, le contenu intentionnel de leurs états et actions est le produit de leur propre activité, et non celle de du théoricien interprétant cette activité. Aussi longtemps que leur intentionnalité est dérivative - parce que la signification normative de leurs états est instituée par l'attitude adoptée à leur égard — leur intentionnalité dérive de chacun, et non de l'extérieur de la communauté. Pour cette raison, seulement des communautés, et non des individus, peuvent être comprises comme possédant une intentionnalité originaire (p. 61).

Je voudrais tenter de montrer que cette solution au problème des " origines de l'intentionnalité » constitue la base d'une solution au problème des « origines de la normativité. » Mais d'abord, quelques mots au sujet du fonctionnement de la solution du problème de l'intentionnalité.

Lorsque nous adoptons une position intentionnelle par rapport à un système quelconque, par exemple un ordinateur jouant aux échecs, nous lui attribuons un ensemble de croyances et de désirs. En d'autres mots, nous essayons d'anticiper ses mouvements en assumant qu'il " essaie de gagner ", et nous ne nous soucions pas du détail de sa programmation. Dans ce cas,

5. C'est en tout cas ce que pense Brandom. Cf. p. 58. 
l'intentionnalité du programme est clairement dérivée. C'est en autant que nous le traitons comme possédant des buts que nous pouvons dire de lui qu'il les poursuit.

Toutefois, cette interaction est caractérisée par une asymétrie significative. Lorsque je joue contre un ordinateur, je suis capable d'adopter une attitude intentionnelle envers lui, mais j'assume qu'il est incapable de faire la même chose envers $\mathrm{moi}^{6}$. Que se passe-t-il si l'interaction est symétrique ? Supposons que l'ordinateur soit remplacé par un opposant que je peux raisonnablement considérer être un système intentionnel interprétant. Une fois cette substitution effectuée, je dois traiter mon opposant, non seulement comme possédant des croyances et des désirs, mais aussi comme m'attribuant des croyances et des désirs. Et si je pense que cet opposant me traite non seulement comme un système intentionnel simple, mais aussi comme un système intentionnel interprétant, alors certaines des croyances qu'il m'attribuera seront des croyances à propos de ses propres croyances, et croyances à propos de ses croyances concernant mes croyances, etc. Ainsi, l'attribution réciproque d'intentionnalité génère une régression de croyances qui augmente considérablement la complexité de l'interaction.

Une façon d'exposer la différence qu'impose cette augmentation de la complexité consiste à observer que les opérations des systèmes intentionnels simples peuvent être modelés à l'aide d'une théorie de la décision, alors que les opérations de deux systèmes intentionnels interprétant, interagissant entre eux, doivent être modelées à l'aide d'une théorie des jeux. Dans un problème décisionnel, l'agent est capable de d'abord former des croyances, puis de sélectionner une stratégie maximisant l'utilité. Par conséquent, il y aura toujours une seule réponse correcte à tout problème. Dans un jeu, les agents ne peuvent pas commencer par arrêter toutes leurs croyances, et souvent ne peuvent faire mieux que de chercher des ensembles de croyances et de stratégies qui sont cohérentes les unes avec les autres. Par conséquent, les problèmes de choix stratégiques peuvent être indéterminés — ils peuvent ne pas avoir une seule réponse correcte ${ }^{7}$.

Deux éléments ressortent ici. Premièrement, il est évident que, dans un certain sens, les états intentionnels de ces deux systèmes sont établis par leur adoption d'une position intentionnelle réciproque. La régression de l'intentionnalité « interprétante » est close — chacun attribue une intentionnalité aux autres, attribue à tous les autres le fait d'attribuer une intentionnalité à tous les autres, et ainsi de suite. Deuxièmement, il y a le fait que cette explication rende

6. En fait, c'est pour cela qu'il est important pour le succès de ces programmes que le jeu d'échecs soit un problème de décision fini - en principe, il y a toujours un meilleur coup. Dans toute partie possédant une dimension stratégique significative - comme le poker - les ordinateurs ont beaucoup plus de difficultés, précisément parce qu'ils sont incapables de modeler les états mentaux de leur opposant avec une certaine sophistication.

7. Pour une discussion plus complète de ces questions, voir Heath, Joseph, "The Structure of Normative Control », Law and Philosophy, 17, 1998, pp. 419-442. 
une certaine forme de réductionnisme impossible. L'attribution mutuelle d'états intentionnels génère une indétermination caractéristique dans le comportement des systèmes intentionnels interprétants lorsqu'ils interagissent les uns avec les autres (indétermination formellement reconnue dans le fait que les interactions stratégiques - les jeux — peuvent avoir des équilibres multiples). Ainsi, alors que l'adoption d'une position intentionnelle à l'égard d'un ordinateur jouant aux échecs est clairement optionnelle, dans le cas de deux systèmes intentionnels interprétants interagissant ensemble, un observateur manquerait clairement quelque chose s'il ne les considérait pas comme des systèmes intentionnels. Le fait qu'ils sont des systèmes intentionnels interprétants fait partie de ce qui les fait agir comme ils le font.

\section{6.}

L'analogie entre le problème de l'intentionnalité et le problème de la normativité est assez apparente. Le problème général avec les théories de la régularité est que n'importe quel segment de comportement peut être dit conforme à un nombre arbitrairement large de règles. Par conséquent, une norme doit être une structure qui est d'une certaine manière " privilégiée » dans ce comportement. La question est alors de déterminer ce qui permet de choisir une structure particulière, l'élevant au-dessus des autres, faisant d'elle la chose correcte à faire, par opposition à seulement la chose qui est faite.

Il est important de noter que, selon Brandom, les sanctions réussissent à résoudre ce problème élémentaire. Une fois que les sanctions sont appliquées à un certain segment de comportement, cela a pour effet de privilégier une structure. On est alors en droit d'affirmer que, dès lors qu'elle est sanctionnée, il existe une norme implicite dans la structure initiale de comportement. Par conséquent, selon Brandom, l'insuffisance de la "solution sanction » ne s'explique pas par le fait que les sanctions sont d'une façon ou d'une autre inappropriées ou insuffisantes. Le problème est que l'application des sanctions génère un problème de régression. Il peut devenir légitime de décrire quelques-unes des actions d'un premier agent comme correctes ou incorrectes en vertu des sanctions d'un second agent. Mais, afin qu'il existe une norme, il faut qu'il y ait une règle quelconque dont les sanctions du deuxième agent constituent l'application. Et, comme on peut dire que l'acte de sanctionner du deuxième agent consiste à appliquer un nombre arbitrairement grand de règles, il doit y avoir quelque chose qui privilégie une de ces structures par-dessus toutes les autres. Mais, si on introduit un troisième agent dont les sanctions privilégieront une certaine structure dans les actions du deuxième, on initie alors une régression.

Ainsi, de la même façon qu'un système intentionnel interprétant confère de l'intentionnalité au système intentionnel simple (comme l'ordinateur jouant aux échecs), un système de sanctions confère de la normativité à une structure de comportement. La régression commence lorsque quelqu'un demande d'où 
provient l'intentionnalité du système intentionnel interprétant, ou encore d'où provient la normativité du système sanctionnant. Ainsi, la «solution sanction " du style d'Haugeland explique seulement le mécanisme par lequel la normativité est héritée. La source continue de nous échapper.

Suivant la logique de Searle, on pourrait être tenté d'affirmer que cette régression crée le besoin d'une structure de comportement possédant une " normativité intrinsèque » - une structure capable de se choisir elle-même comme normativement privilégiée. (C'est, par exemple, la stratégie que suit Christine Korsgaard dans sa quête de la source de la normativité ${ }^{8}$.) Toutefois, de la même manière qu'il veut maintenir la " posture interprétative» dans sa discussion de la normativité, Brandom veut aussi maintenir l'idée que les statuts normatifs sont en bout de ligne constitués par des attitudes et des évaluations normatives. Il ne peut donc pas considérer que quelque chose est normatif de façon inhérente. Par conséquent, sa tendance est simplement d'affirmer que la régression de la normativité est sans conséquence ou à tout le moins non pertinente, étant donné que nous habitons déjà l'espace de la normativité. C'est cela qui est sous-jacent à son affirmation que nos pratiques sont «tout en normes ».

Une solution beaucoup moins problématique est toutefois suggérée par l'approche qu'adopte Brandom face au problème de l'intentionnalité. Rappelons comment la régression surgit. Supposons qu'une personne agit. Afin de dire que cette action est conforme à la règle, nous devons introduire une seconde personne qui sanctionnera la première. Et afin de dire que cette sanction est conforme à la règle, nous devons faire intervenir une troisième personne qui sanctionnera la seconde, et ainsi de suite, du moins, à ce qu'il semble. Mais devons-nous introduire la troisième personne?

Une manière aisée d'éliminer la régression est simplement de fermer le cercle après la première itération. Au lieu d'introduire une troisième personne pour sanctionner la seconde, nous pouvons simplement stipuler que la première personne sanctionne la seconde. Le second agent possède ce qui peut être appelé une " attente de comportement » - il s'attend à ce que la première personne agisse d'une certaine façon. Si la première personne anticipe ces attentes, elle peut développer ce que nous pouvons appeler une " attente de reconnaissance »- elle s'attend à ce que la seconde personne réponde correctement à ses actions, qu'elle le punisse seulement lorsqu'il est approprié de le faire, ou qu'elle le récompense lorsqu'elle est en droit de l'être'. Lorsque l'une ou l'autre des attentes est déçue, des sanctions sont imposées. Ainsi, les efforts de sanction de la seconde personne deviennent

8. Korsgaard, Christine M. The Sources of Normativity, Cambridge, Cambridge University Press, 1996.

9. Cette terminologie, comme l'idée générale qui sous-tend cette analyse, provient de Habermas, Jürgen, The Theory of Communicative Action, Vol. 2, Boston, Beacon Press, 1987, p. 19. 


\section{Philosophiques / Printemps 2001}

sujets aux sanctions de la première, de la même façon que les actions de la première personne sont sujettes aux sanctions de la seconde.

Évidemment, cette structure de sanctions et d'attentes réciproques génère sa propre forme de régression. La manière dont la première personne sanctionne les efforts de sanction de la seconde doit aussi être sanctionnée par la seconde. Mais, cette forme de régression est clairement sans conséquence dans les cas où attentes et sanctions convergent vers une même structure d'action. Or, ces cas sont précisément ceux dans lesquels on voudrait dire qu'il existe une norme implicite dans la pratique. Lorsque la seconde personne s'attend à ce que la première fasse $x$, la première s'attend à ce que la seconde s'attende à ce qu'elle fasse $x$, la seconde s'attend à ce que la première s'attende à ce qu'elle s'attende à ce que la première fasse x, etc., et lorsque toutes ces attentes sont supportées par des sanctions, alors seulement une action, c'est-à-dire $x$, peut satisfaire toutes ces attentes. Ainsi, $x$ est l'action correcte. Par conséquent, la régression, loin d'être vicieuse, génère quelque chose de très semblable à un ensemble d'attentes se renforçant mutuellement pour soutenir des équilibres jeu-théorétiques.

Par conséquent, lorsque l'acte de sanctionner est réciproque, deux agents peuvent chacun agir d'une manière qui confère de la normativité aux actions de l'autre et cela, par extension, confère de la normativité à leurs propres actions. Il n'y a rien de plus, dans l'interaction, qui puisse compter comme un "simple comportement ». En particulier, parce que chacun s'engage dans une évaluation normative de la conduite de chacun, chacun n'a d'autre choix que d'adopter (au moins implicitement) une évaluation normative de sa propre conduite. Ainsi, il est plausible de suggérer qu'une " normativité originaire » est inhérente aux pratiques d'une communauté dans laquelle chaque personne sanctionne chacune des autres (et il n'importe nullement que ces sanctions soient « internes » ou « externes »).

\section{7.}

Cette explication de la normativité originaire possède deux caractéristiques qui la rendent intéressante du point de vue de Brandom, et qui sont intimement reliées. Premièrement, selon cette conception, la normativité n'est pas simplement une façon de parler ${ }^{10}$. Le vocabulaire n'est pas une composante optionnelle de nos discussions de l'interaction sociale. Chaque fois que nous avons un ensemble d'attentes normatives se soutenant mutuellement, la seule manière de comprendre correctement la structure d'action qui en résulte devra être exprimée en termes de ces attentes. La seconde caractéristique intéressante de cette explication est qu'elle fait de l'interaction normative une sorte de structure d'interaction sui generis et satisfait ainsi la préoccupation 
de Brandom qu'on ne doit entretenir « aucune réduction de statut normatif à des dispositions non-normativement spécifiables. »

Prenons ces deux éléments dans l'ordre.

Lorsque deux individus interagissent d'une façon authentiquement normative, en accord avec l'explication présentée ci-dessus, alors un observateur manquerait clairement quelque chose s'il n'interprétait pas cette conduite en termes normatifs. Considérons encore une fois l'analogie avec la théorie du jeu. Imaginons une interaction entre deux joueurs possédant plusieurs équilibres Nash, et supposons qu'un de ces équilibres soit joué. Cela veut dire que l'action de chacun des joueurs constituera une meilleure réponse à l'action de l'autre. Ainsi, si nous demandons pourquoi le joueur 1 a choisi $x$, la réponse sera qu'il s'attendait à ce que le joueur 2 choisisse $y$. Si nous demandons pourquoi le joueur 2 a choisi $y$, la réponse sera qu'il s'attendait à ce que le joueur 1 choisisse $x$. Par conséquent, l'attente du joueur 1 que le joueur 2 choisira $y$ est fondée dans l'attente du joueur 2 que le joueur 1 choisira $x$, et vice-versa. Le fait que ces deux ensembles d'attentes se renforcent mutuellement est la seule chose qui soutienne la structure de comportement (étant donné que, $e x$ hypothesi, aucune des actions n'est intrinsèquement valable). Par conséquent, la seule manière de comprendre l'interaction est de saisir cet ensemble sousjacent de croyances et d'intentions.

Comprendre une interaction normativement régulée impose les mêmes types d'exigences. Imaginons une règle simple spécifiant que $x$ est normativement requis du joueur 1 . Une telle règle peut être établie par une structure de sanction qui assigne au joueur 2 une action $y$, qui est conditionnelle à l'exécution de l'action $x$ par le joueur 1. L'ensemble des attentes normatives peut alors être clos en faisant en sorte que, dans les itérations subséquentes de l'interaction, l'obligation du joueur 1 de faire $x$ soit conditionnelle à l'exécution appropriée de l'action $y$ par le joueur 2 en réponse à $x$. Alors, il sera vrai que le joueur 1 a l'intention de faire $x$ parce qu'il s'agit de la réponse appropriée à $y$, et que le joueur 2 a l'intention de faire $y$ parce que c'est la réponse appropriée à $x$. Un observateur qui ne noterait pas cette structure sous-jacente d'attentes normatives manquerait encore une fois quelque chose, étant donné que ces attentes sont constitutives de la structure que l'interaction expose.

L'autre élément méritant d'être noté est que, dans une interaction régulée normativement, aucun des joueurs n'a besoin d'avoir une quelconque disposition concrète à exécuter une action spécifique. Le joueur 1 peut ne pas avoir de raison particulière de choisir $x$ autre que le désir de forcer le joueur 2 à choisir $y$, et le joueur 2 peut ne pas avoir de raison particulière de choisir $y$ autre que le désir de forcer le joueur 1 à jouer $x$. Par conséquent, il peut être impossible d'expliquer la structure d'interaction en termes d'un certain ensemble de dispositions de comportement antérieurement déterminées, parce qu'une telle explication manquerait d'expliquer l'élément crucial, soit comment $x$ est tout d'abord devenu le point focal de ces attentes normatives. 


\section{Philosophiques / Printemps 2001}

L'illustration la plus claire de cette thèse peut être vue dans le fait que l'émergence de structures d'interaction authentiquement normatives impose des limites à l'étendue des comportements humains qui peuvent être expliqués en utilisant la théorie darwiniste standard de l'évolution. La raison à cela - en résumant trop brièvement une littérature relativement complexe - est que les interactions régulées normativement créent la possibilité d'une transmission culturelle du comportement appris. Cela veut dire que notre comportement, contrairement à celui d'autres animaux, doit être expliqué en utilisant un modèle de "double héritage ", dans lequel on explique les contributions des facteurs génétiques et culturels en utilisant des modèles de transmission différents ${ }^{11}$.

Tous les organismes ont une certaine capacité à modifier leur phénotype en réponse aux caractéristiques de leur environnement. Alors que certains des éléments de ce phénotype font génétiquement partie du hardwire, plusieurs autres sont appris. Dans sa forme la plus simple, apprendre requiert seulement un mécanisme qui fera que l'animal sera plus enclin à exécuter des actions qui ont réussi par le passé. Ainsi, deux organismes possédant le même génotype peuvent montrer des phénotypes différents en fonction des aspects de l'environnement qui affectent leur développement.

Lorsqu'un tel mécanisme d'apprentissage est en place, il devient possible d'utiliser des sanctions afin d'inculquer des routines de comportement. C'est ce qu'on nomme l'apprentissage social. Une telle capacité permet aux espèces de reproduire fidèlement certaines caractéristiques phénotypiques, même lorsqu'elles ne sont pas directement spécifiées dans le génotype. Toutefois, il y a des limites au phénotype parce que le comportement-sanction, qui génère ultimement les effets phénotypiques, doit encore être spécifié dans le génotype afin qu'il soit fidèlement reproduit.

Toutefois, lorsque le comportement-sanction devient lui-même sujet à des sanctions, il devient possible pour le phénotype de s'écarter arbitrairement du génotype. Il peut alors se produire ce que Boyd et Richerson appellent les «effets niveau-population » ${ }^{12}$. De nouveaux phénotypes peuvent apparaître et être "transmis ", sans égard à la structure génétique sousjacente. C'est l'évolution culturelle. Parce que ce comportement n'est pas seulement transmis "verticalement »(du parent à l'enfant), mais aussi " horizontalement " (parmi les frères et sœurs, les pairs, les congénères), les pressions sélectives fonctionneront d'une manière bien différente de celle dont elles fonctionnent dans le domaine de la génétique.

Que veut dire tout cela ? Les tendances comportementales génétiquement déterminées suffisent pour expliquer un très grand nombre de comportements animaux, même de types très complexes. Ces tendances peuvent

11. Je m'inspire ici de Boyd, Robert et Richerson, Peter J., Culture and the Evolutionary Process, Chicago, University of Chicago Press, 1985.

12. Ibid., p. 6. 
aussi expliquer des comportements de socialisation; elles peuvent ainsi expliquer indirectement des éléments du phénotype qui sont acquis au moyen de l'apprentissage social. Toutefois, lorsque le comportement de socialisation devient lui-même l'objet de la socialisation (par exemple, lorsque le cercle de la sanction se referme), ce type d'explication se heurte à une limite de principe. Des formes phénotypiques peuvent apparaître et être transmises sans aucune base génétique sous-jacente. Par conséquent, le normatif devient une composante irréductible de notre économie comportementale, et une source de formes phénotypiques et d'effets de niveau-population sui generis.

Ce point a des conséquences extrêmement importantes. Il y a un courant influent du pragmatisme, peut-être formulé de la manière la plus influente par Richard Rorty, qui est enclin à penser qu'ultimement, on expliquera les composantes comportementales de la théorie pragmatiste par rapport à une certaine théorie de l'évolution naturelle. Comme Rorty l'exprime :

Ce que je retiens, c'est la conviction que le darwinisme procure un vocabulaire utile pour formuler la position pragmatiste (...). Par « darwinisme ", j'entends une histoire au sujet des humains compris comme des animaux possédant des organes et capacités particuliers : au sujet de la manière dont certaines caractéristiques de la gorge, des mains, des cerveaux humains ont rendu les humains capables de commencer à développer des pratiques sociales de plus en plus complexes en générant des sons de plus en plus complexes. Selon cette histoire, ces organes et capacités et les pratiques qu'ils rendent possibles sont pour beaucoup dans ce que nous sommes et ce que nous voulons, mais ils ne nous mettent pas plus en relation de représentation avec une nature intrinsèque des choses que ne le fait le museau du tamanoir ou le talent de certains oiseaux au tissage. ${ }^{13}$

Si on souscrit à cette conception des « hommes-comme-animaux-unpeu-plus-compliqués » ${ }^{14}$, alors il n'y a aucune raison d'assigner à la normativité une position très importante dans l'ordre global de l'explication pragmatiste (aussi longtemps, à tout le moins, que l'on veut nier que les animaux s'engagent dans des pratiques authentiquement normatives). Ainsi, Brandom a de la difficulté à rejeter l'idée selon laquelle la différence entre l'intelligence humaine et animale n'est qu'une question de degré, et que nos pratiques ne diffèrent que par le fait que nous démontrons un niveau de complexité plus élevé. (C’est, par exemple, une des raisons qu'il invoque pour distinguer entre intelligence et sapience). De ce point de vue, une conception comme celle de Rorty est un type de réductionnisme.

L'explication de la normativité dont j'ai exposé les grandes lignes cidessus est conçue pour procurer un fondement à l'affirmation de Brandom

13. Rorty, Richard, "Putnam and the Relativist Menace ", Journal of Philosophy, 90, 1993, pp. 443-61, aux pages 447-8.

14. Ibid, p. 448. 


\section{Philosophiques / Printemps 2001}

selon laquelle il existe une distinction de principe à tracer entre des pratiques sociales régies par des règles et d'autres formes de comportements sociaux complexes. En même temps, cela montre comment une forme grossière de réductionnisme darwiniste - une forme qui ne reconnait pas la contribution distinctive que la régulation normative de la conduite apporte à la structure des interactions sociales humaines - sera sujette à des déficiences explicatives et prédictives. Ainsi, le but de Brandom d'expliquer les statuts normatifs en termes d'évaluations normatives est atteint, mais sans générer une régression vicieuse de la normativité et sans courir un risque de réductionnisme. 